\title{
Wireless Sensing of Discharge Characteristics F OR QUALITy CONTROL IN BATCH MODE MiCRO-ELECTRO-DISCHARGE MACHINING
}

\author{
Mark T. Richardson ${ }^{1 *}$, Ranjit Gharpurey ${ }^{2}$, and Yogesh B. Gianchandani ${ }^{1}$ \\ ${ }^{1}$ Department of Electrical Engineering and Computer Science, University of Michigan, Ann Arbor, MI, USA \\ ${ }^{2}$ Department of Electrical and Computer Engineering, University of Texas, Austin, TX, USA
}

\begin{abstract}
Wireless signals are inherently generated during the spark discharges of micro-electro-discharge machining ( $\mu \mathrm{EDM})$. This work harnesses signals between $300-750 \mathrm{MHz}$ for the monitoring of batch mode machining quality. In particular, the wireless spectra are used to sense incipient, debris-dominated machining that can be destructive to both the tool and the workpiece. Planar stainless steel $(316 \mathrm{~L})$ is machined $50 \mu \mathrm{m}$ deep in a high-density stent pattern with $30 \mu \mathrm{m}$ features and tool fill factors ranging from $8.4 \%$ to $32.9 \%$. In debris-dominated conditions, the $580-750 \mathrm{MHz}$ frequency band shows an increased average strength over an average reference band of $410-480 \mathrm{MHz}$. The temporal evolution of the spectra permits the discharges to be monitored in a manner that is less affected by circuit parasitics than alternative electrical methods. It is amenable to real-time monitoring of large-scale production.
\end{abstract}

\section{INTRODUCTION}

Micro-electro-discharge machining $(\mu \mathrm{EDM})$ is a technique that utilizes spark discharges between a tool and workpiece in a dielectric fluid to micro-machine any conductive material [1]. By finely controlling the discharge energy as well as the positioning of the tool and workpiece, any arbitrary shape can be machined. Recent applications include a cardiovascular antenna stent (stentenna) [2,3] (Fig. 1), RF switches [4], and DC-to-DC boost converters [5].

Traditionally, serial mode $\mu \mathrm{EDM}$ uses a sharpened wire as the tool, which is scrolled across the workpiece. Batch mode $\mu \mathrm{EDM}$ uses a cookie cutter type tool to machine the entire pattern in parallel. The advantages are that batch mode is lithographically

A)
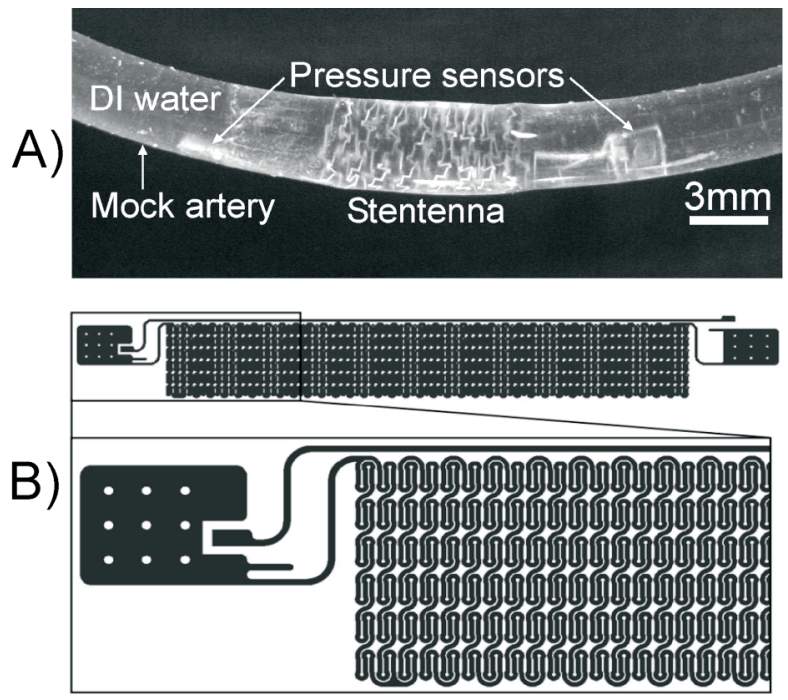

Fig. 1: (A) Low-Q flow sensing Stentenna in mock artery [2]. (B) High-Q Stentenna pattern for batch $\mu$ EDM. Solid sections are pads for mounting pressure sensors [3].

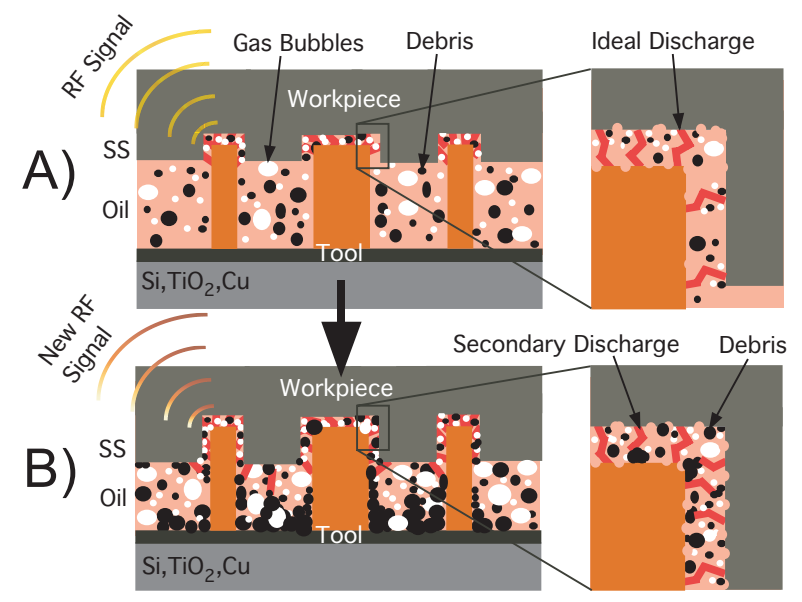

Fig. 2: (A) Ideal $\mu E D M$ spark discharges produce a wireless signal that changes with secondary discharges (B) due to debris.

compatible and offers a 100x improvement in throughput over serial mode [6]. Batch mode tools can be fabricated using PMMA for X-ray or SU-8 for UV exposure in a LIGA type electroforming process. One drawback is that as feature densities increase, accumulation of trapped debris leads to spurious, secondary discharges. Without attention, this causes workpiece damage and tool recasting, eventually halting progress completely (Fig. 2) $[7,8]$. Damage to the workpiece consists of rough surfaces, rounded edges, and larger machining tolerances. Tool recasting is caused by secondary discharges that lead to excessive heating. The result is a tool that is wider and shorter than the original at different locations on the pattern. It would be useful to detect the change in machining conditions before this damage occurs.

Fast current spikes generate a detectable wireless signal [911]. Marconi first utilized spark discharges similar to those found in EDM for wireless communication in the 1890s. In the late 1970s, early work showed that it was possible to use RF transmissions to distinguish between open circuit, spark, arc, and short circuit conditions in macro-scale, serial mode EDM [12]. At the micro scale, particularly for batch mode, process monitoring is even more critical. Further, due to the smaller dimensions, tighter tolerances, electrode multiplicity and lower machining rates, the discharge characteristics are significantly different.

As debris accumulation becomes worse during machining, one would expect changes to the discharges and consequently, the wireless spectrum. In this study we explore how these characteristics can be correlated to micromachining process quality with a view toward monitoring large-scale production by $\mu \mathrm{EDM}$.

\section{EXPERIMENT DEFINITION}

The test pattern used for this study was the stentenna [2,3]. Figure 1A shows a low-Q stentenna fabricated with serial $\mu \mathrm{EDM}$ that has been deployed in a mock artery. The device begins as a planar stainless steel foil that is machined into a meandering

*Corresponding Authors: 1301 Beal, Ann Arbor, MI 48109, mtrichar@umich.edu, yogesh@umich.edu 
pattern. The coil is an inductor and the pressure sensors are capacitors in an LC tank. This allows wireless querying of the sensors for in-situ flow measurement in an artery by tracking the resonant frequency of the LC tank.

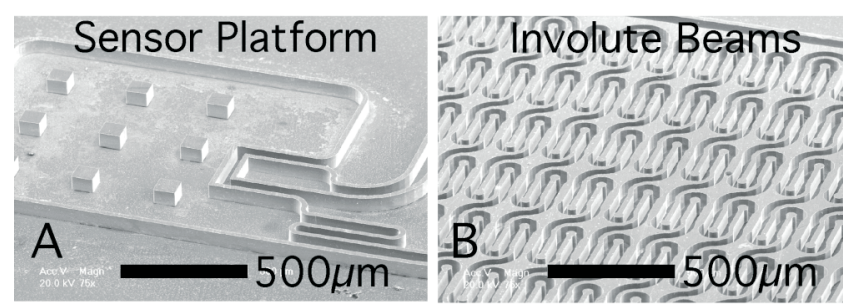

Fig. 3: $80 \mu \mathrm{m}$ copper electroplated high-Q stentenna tool. Left: sensor platform. Right: Involute beams [3].

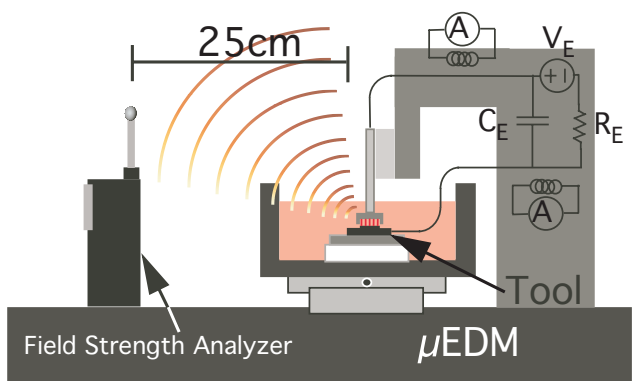

Fig. 4: Experiment setup for wireless monitoring and inductively coupled current probes.

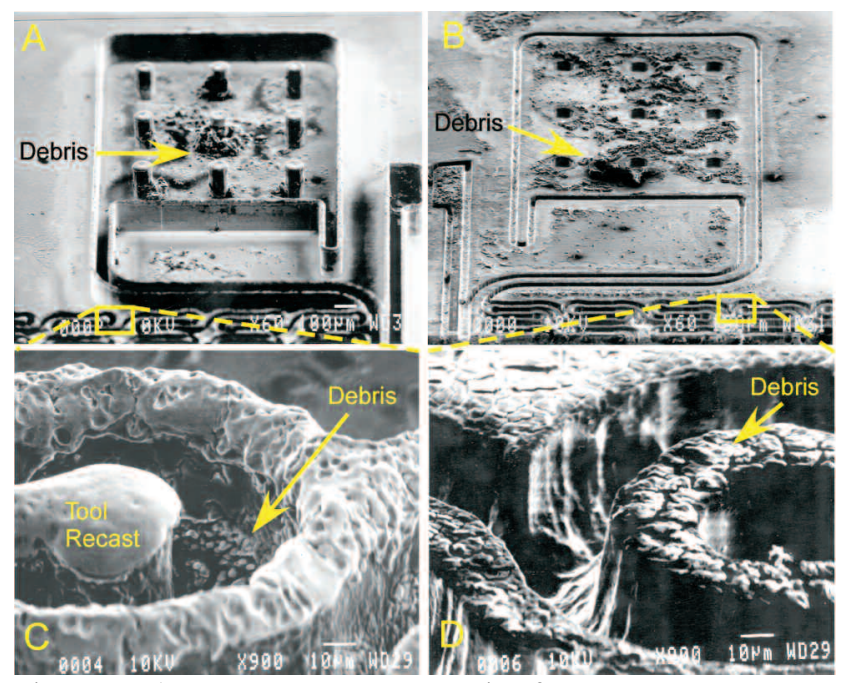

Fig. 5: A) $200 \mu \mathrm{m}$ copper tool after machining with debris. B) Workpiece with large debris before cleaning. C) Close-up of debris, tool recast. D) Debris on workpiece top before cleaning, damaged sidewalls. E) Straight edges from non debris-dominated $\mu \mathrm{EDM}[8]$.

Table I: Machining Conditions

\begin{tabular}{|l|c|}
\hline Tool Height & $200 \mu \mathrm{m}$ \\
\hline Voltage & $70 \mathrm{~V}$ \\
\hline Capacitor & $10 \mathrm{pF}$ \\
\hline Resistor & $5 \mathrm{k} \Omega$ \\
\hline Z-Feed & $0.2 \mu \mathrm{m} / \mathrm{s}$ \\
\hline Plunge & $50 \mu \mathrm{m}$ \\
\hline Workpiece & $316 \mathrm{~L} \mathrm{Steel}$ \\
\hline
\end{tabular}

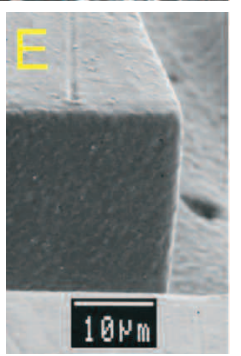

While this new functionality is promising, the quality factor (Q) of the LC tank significantly limits the range and sensitivity. Three ways that the $\mathrm{Q}$ can be improved are to increase the inductance, decrease the parasitic resistance, or lower the nominal capacitance of the pressure sensors [3]. High-density batch mode $\mu \mathrm{EDM}$ enables the number of inductor turns to be greatly increased, giving a 16x potential improvement in inductance. This is implemented in the high-Q stentenna pattern shown in Figs. 1B and 3. The pattern is $2.5 \mathrm{~cm}$ long and $2.5 \mathrm{~mm}$ wide with $30 \mu \mathrm{m}$ wide beams. However, due to the compact, meandering shape, this pattern is hindered by debris accumulation [3] and is a good test for the wireless monitoring technique.

The pattern can be divided into two sections, the sensor platform and the involute beams. The sensor platform (Fig. 3A) has a fill factor (tool area density) of $8.4 \%$. The involute beams (Fig. 3B) have a fill factor of $32.9 \%$. Copper tools were fabricated using $200 \mu \mathrm{m}$ tall PMMA LIGA on a titanium oxide seed layer. The workpiece was a $7 \times 7 \mathrm{~mm}^{2}, 100 \mu \mathrm{m}$ thick $316 \mathrm{~L}$ stainless steel foil, the same material as commercial stents. A small portion of the pattern was machined with minimum discharge energy to allow longer observation of the spectra as debris accumulated. In Fig. 4, which shows the machining apparatus, this translated to values of $70 \mathrm{~V}$ and $10 \mathrm{pF}$ for $\mathrm{V}_{\mathrm{E}}$ and $\mathrm{C}_{\mathrm{E}}$, respectively.

In serial mode, for which the tool is a wire tip, the tool is rapidly rotated to improve dimensional accuracy. This also helps remove debris by continuously flushing the dielectric fluid. In batch mode, since the tool is a 2-dimensionial cookie cutter, it cannot be rotated. Instead, the stage is dithered vertically by about $20 \mu \mathrm{m}$ during machining to flush the fluid as much as possible. Machining parameters are listed in Table I. The sample was plunged $50 \mu \mathrm{m}$ deep into the tool while recording the wireless RF field strength, plunge depth, time, and current waveform of the discharges. Note that plunge depth indicates how far the tool has advanced into the workpiece during machining. If there is appreciable tool wear, the actual machined depth may be somewhat less.

\section{EXPERIMENTAL RESULTS}

The SEM images in Fig. 5 show the tool and workpiece after machining. There was enough residual debris on the surface to span the full height of the tool. A significant amount of debris was left in the dielectric oil that could not be imaged. Figs. 5C and 5D show tool recasting and debris on the surfaces, indicating a large amount of debris near the machining area. Figure $5 \mathrm{E}$ shows a different example of high quality machining with no debris effects [8]. In Fig. 6, one can see that machining progressed steadily until 165 min. when machining became debris-dominated. Changes in discharge characteristics (and consequently the RF transmission)

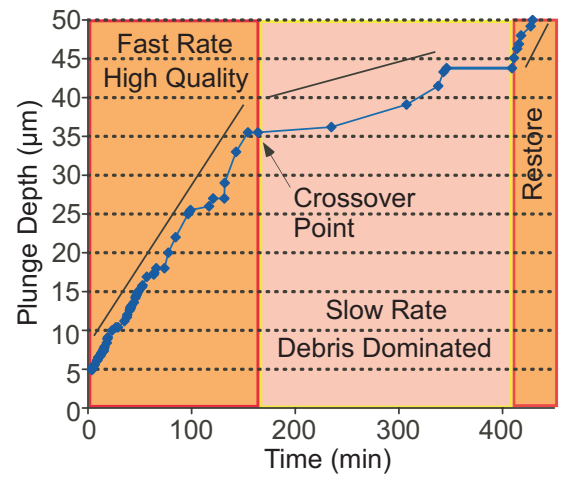

Fig. 6: Plunge rate slows around $165 \mathrm{~min}$ and restores around 410 min. This is because discharges are used to pulverize debris and the workpiece repeatedly retracts when it senses such events. 


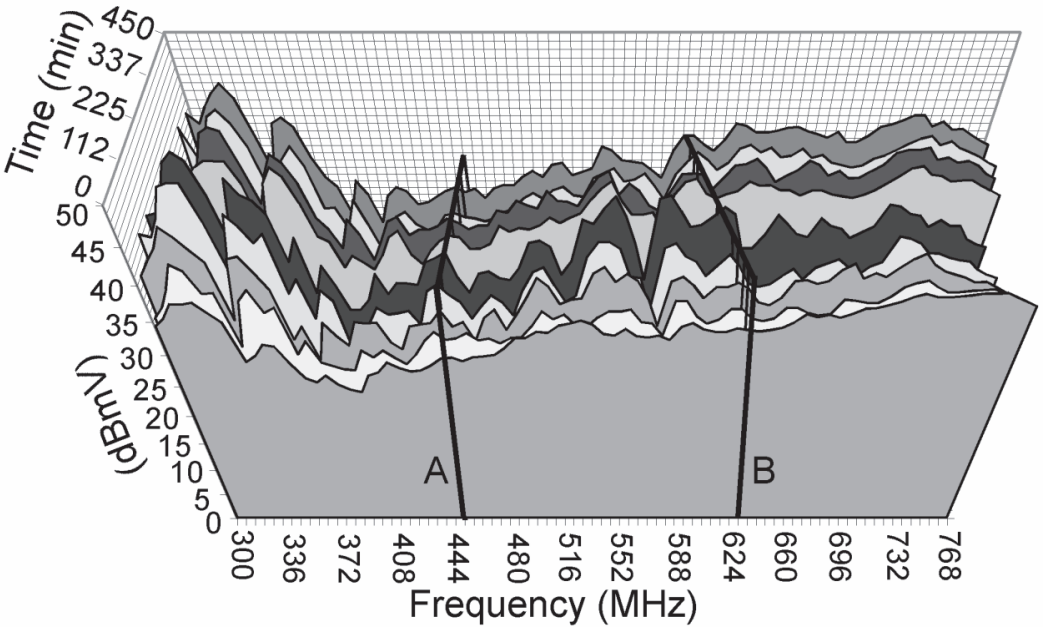

Fig. 7: Wireless signal strength at a distance of $25 \mathrm{~cm}$ for various times and frequencies. Each spectrum represents the average of 10 samples. Half the number of samples shown for clarity. Frequencies A and B follow different trends when normalized.

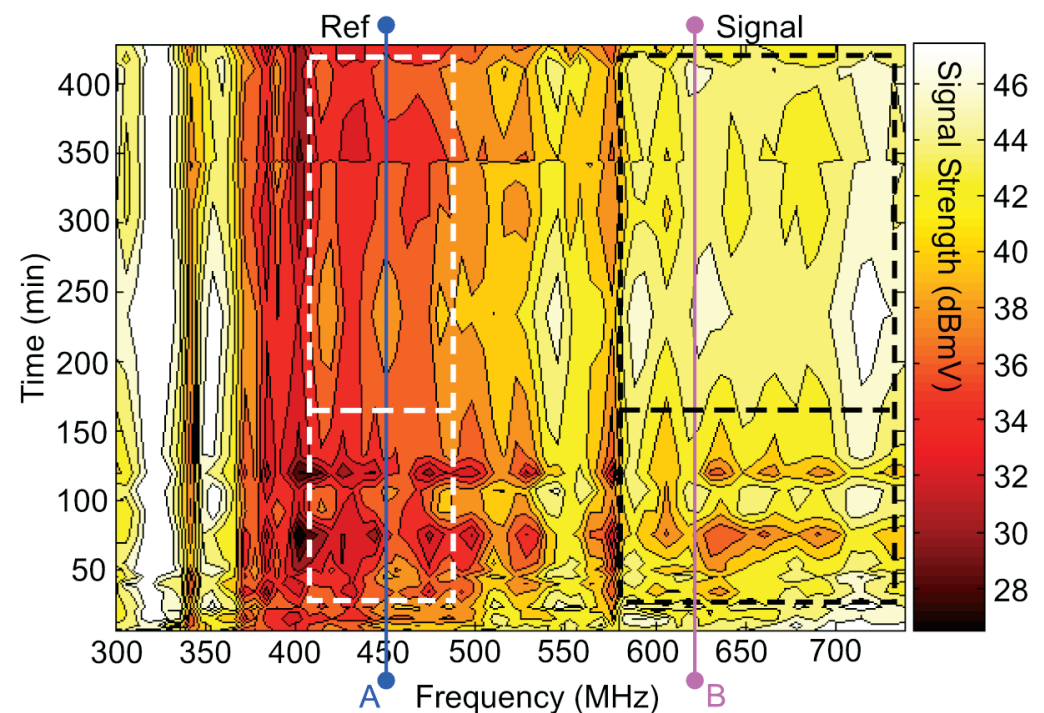

Fig. 8: Topology view (top view) of wireless signal strength over the course of machining. Each spectrum is normalized to the highest peak and plotted in Matlab. Frequencies A and B in the Ref and Signal bands change strength at different rates.

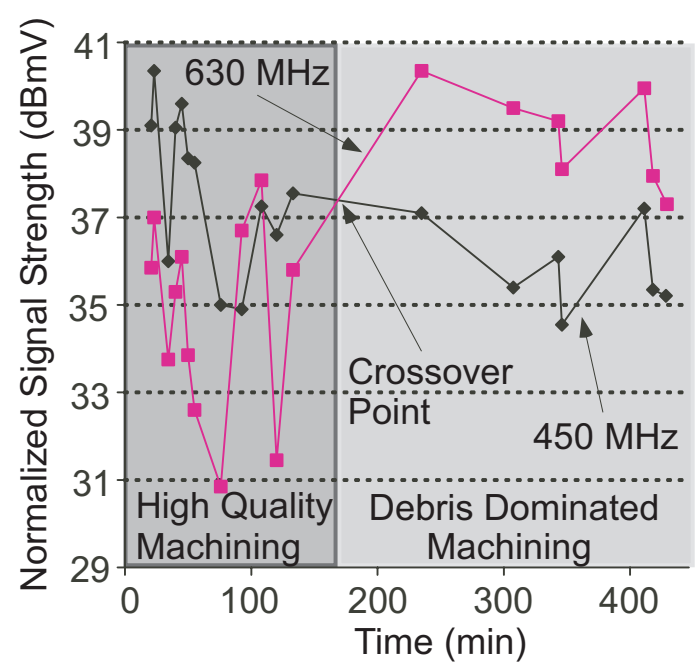

Fig. 9: The two normalized frequencies from Fig 8. A is a reference for $\mathrm{B}$. Note opposing trend at $\sim 132 \mathrm{~min}$, right before the plunge rate slow down in Fig 6. Also at $100 \mathrm{~min}$. First 3 data points not shown for clarity.

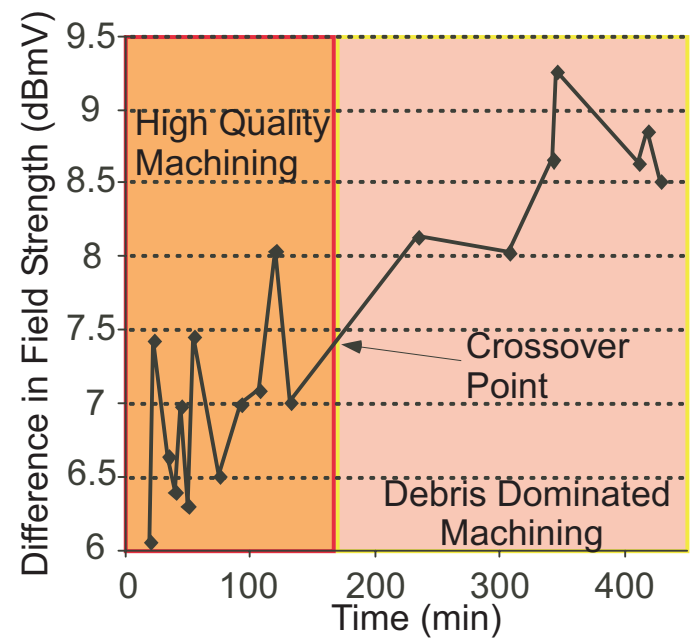

Fig. 10: The difference of the averages of the Signal and Ref bands in Fig. 8 eliminates common amplitude variations over time. Small numbers match favorable machining condition while larger numbers match poor machining according to plunge rates in Fig. 6. are anticipated at such transition points. The potential advantage of monitoring the RF signals is that they are directly indicative of the nature of the spark. In batch mode machining, the measurable electrical characteristics of the discharge pulse are affected by a variety of parasitics. Hence, they are not always the best indicator of real-time machining quality.

Wireless measurements were taken at a distance of $25 \mathrm{~cm}$ using a Protek 3201 field strength analyzer (Fig. 4). The spectrum plotted in Fig. 7 was recorded over time from 300 to $750 \mathrm{MHz}$ in 6 $\mathrm{MHz}$ steps. Data points represent the average of 10 samples and were compensated for antenna factor and background noise. The frequency range was selected to cover the upper bound of the signal and detect frequency shifts in that range. In Fig. 8, the topology view (top view) of the same data is normalized to the highest peak in each spectrum and plotted against time on the yaxis. It is a Matlab interpolation of the data points that helps distinguish trends in the signal over time. There are two regions of interest that show different trends, 'Ref' and 'Signal'. From Fig. 8 it is clear that frequencies from $580-750 \mathrm{MHz}$ can be used as an averaged indicator for the crossover to debris-dominated machining. Frequency bands in the range $410-480 \mathrm{MHz}$ provide a reasonable average reference for comparison.

The maximum output signal is represented by the 450 and 630 $\mathrm{MHz}$ signal lines and can be seen more clearly in Fig. 9. The 450 $\mathrm{MHz}$ line acts as a reference for the $630 \mathrm{MHz}$ line. These frequencies follow the same signal strength trend until $132 \mathrm{~min}$., where the $630 \mathrm{MHz}$ line becomes larger ultimately crossing the $450 \mathrm{MHz}$ line at $165 \mathrm{~min}$. (Note that this is consistent with transition of the plunge rate (slope) in Fig. 6 where machining becomes debris-dominated at $165 \mathrm{~min}$.). The maximum difference was $9.3 \mathrm{dBmV}$. Figure 10 plots the difference in the averages of the Signal and Ref. bands as a function of time. Amplitude variations that are common to both frequencies are removed, but a clear trend reflects the progressive accumulation of debris. The smaller regions track the high quality machining from Fig. 6 while larger regions track the debris-dominated machining.

Low impedance current probe traces for the cathode and workpiece in Fig. 11 gave small differences between high quality 
and debris-dominated regions. As noted previously, electrical characteristics measured at the discharge electrodes in this manner are influenced by a number of electrical parasitics and are not directly indicative of the nature of the spark. Wireless measurement, therefore, promises a superior technique for real time monitoring of batch mode $\mu \mathrm{EDM}$ machining quality.

\section{DISCUSSION}

During the $\mu \mathrm{EDM}$ process, as the debris accumulate; they begin to interfere with the discharge gap as diagramed in Fig. $2 \mathrm{~b}$. Inspection of the tool after machining shows that the amount of debris can span the distance from workpiece to tool. It has also been shown that metallic particles can transport charge from one electrode to another before discharging at much shorter distances and smaller energies [13]. These effects lead to detectable changes in the wireless field strength as the level of debris increases.

Spark discharges between two conductors in air have been modeled in the past as dipole radiation to simulate electrostatic discharge effects on circuitry [9-11]. While these models are for gas discharges and spherical electrodes, the trends are still useful to understand since a $\mu \mathrm{EDM}$ discharge still has many similarities. In [10] it was found that as the discharge gap decreased, the discharge voltage decreased, the amplitude of the electromagnetic field intensity increased, and the time rate change of the current increased. The dipole model for a square wave current pulse from $0<\mathrm{t}<\tau_{0}$ is given for the magnetic flux density by:

$$
B \approx-\frac{2 l \sin (\theta)}{c^{2} r} \frac{\partial I(t-r / c)}{\partial t}
$$

where $l$ is gap length, $c$ is the speed of light in the medium, $t$ is time, $\mathrm{r}$ is distance to the observation point at angle $\theta$, and $I()$ is the current waveform [9]. The Fourier components of B are given by:

$b=\frac{4 i \sin \theta e^{i \omega\left[r / c+\tau_{0} / 2-(l / c) \cos \theta\right]}}{c r \cos \theta} \sin \left(\frac{\omega l}{c} \cos \theta\right) \cos \left(\frac{\omega \tau_{0}}{2}\right)$

While direct measure of these terms is not possible, it appears likely that in the context of batch mode $\mu \mathrm{EDM}$, the accumulation of debris influences $\tau_{0}$, leading to an increase in high frequency components of the radiated spectrum. Further investigation is in progress.
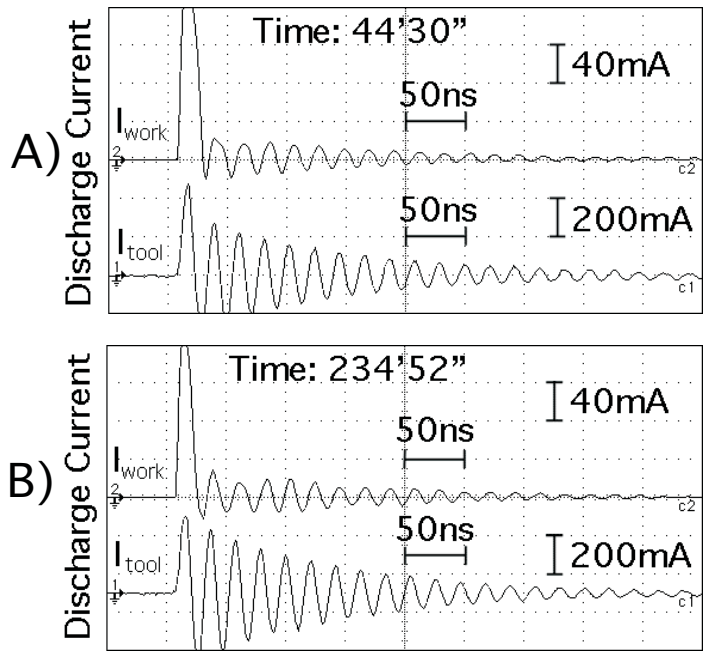

Fig. 11: Current traces for the cathode and workpiece during (A) normal machining and (B) debris-dominated regions of machining do not provide obvious indicators of decreased machining quality.

\section{CONCLUSIONS}

The discharges in $\mu$ EDM produce detectable wireless signals. As debris accumulate in batch mode $\mu E D M$, the signal amplitude at various frequencies changes. In particular, the average transitions at frequencies $>580 \mathrm{MHz}$ gain in strength while others remain relatively constant over time. The difference between either two frequencies in these bands or the averages of these bands tracks the favorable and debris-dominated machining conditions. Wireless monitoring could be used to predict and prevent damage to the workpiece and tool in real time. However, frequency selection would be dependent on materials and machining conditions. Wireless monitoring could be built to interface with the control hardware of a $\mu \mathrm{EDM}$ and used in an automated production context to monitor multiple machines at once.

\section{ACKNOWLEDGEMENTS}

The authors would like to acknowledge Dr. Dawn Skala and Brandon Levey for fabrication assistance as well as Dr. Kenichi Takahata for discussions. The facilities used for this research include Sandia National Laboratories, California and the University of Michigan. Travel support has been provided by the Transducers Research Foundation.

\section{REFERENCES}

[1] T. Masaki, K. Kawata, and T. Masuzawa, "Micro electrodischarge machining and its applications," IEEE MEMS '90, pp. 21-26, 1990

[2] K. Takahata, A. DeHennis, K.D. Wise, Y.B. Gianchandani, "Stentenna: a micromachined antenna stent for wireless monitoring of implantable microsensors," IEEE Conf EMBS, 2003, pp. 3360-3 [3] K. Takahata, Batch manufacturing technology based on microelectro-discharge machining and application to cardiovascular stents. University of Michigan, Electrical Eng Ph.D. Thesis, 2005

[4] K. Udeshi, M. Richardson, J.-J. Hung, L. Que, G.M. Rebeiz, Y.B. Gianchandani, "A dual-EDM reverse damascene process for RF switches and other bulk devices," ASME IMECE, Nov. 2005

[5] K. Udeshi, On-chip high voltage generation using mechanical oscillators. University of Michigan, Mech Eng Ph.D. Thesis, 2005

[6] K. Takahata, Y.B. Gianchandani, "Batch Mode Micro-ElectroDischarge Machining," JMEMS 11(2), pp. 102-110, 2002

[7] M.T. Richardson, Y.B. Gianchandani, "A Passivated Electrode Batch $\mu$ EDM Technology for Bulk Metal Transducers and Packages," IEEE Sensors 2005, pp. 219-222, Nov. 2005

[8] M.T. Richardson, Y.B. Gianchandani, D.S. Skala, "A parametric study of dimensional tolerance and hydrodynamic debris removal in micro-electro-discharge machining," IEEE MEMS 2006, pp. 314-317, Jan. 2006

[9] A. Kadish, W. B. Maier, "Electromagnetic radiation from abrupt current changes in electrical discharges," J. Appl. Phys. 70(11), pp. 6700-11, Dec. 1, 1991

[10] H. Tomita, "Dependence of current and induced voltage due to spark discharge on gap length," Intl Sym on Emag Compatibility, pp. 138-141, 1999

[11] S. Ishigami, T. Iwasaki, "Evaluation of charge transition in a small gap discharge," IEICE Trans. Commun, 79B(4), pp. 474-82 Apr. 1996

[12] S.K. Bhattacharya, M.F. El-Menshawy, "Monitoring the EDM process by radio signals," Intl J.Prod Res, 16(5), pp. 353-63, 1978

[13] R. Tobazeon, "Electrohydrodynamic behaviour of single spherical or cylindrical conducting particles in an insulating liquid subjected to a uniform DC field," J. Phys. D: Appl. Phys., 29, pp. 2595-2608, 1996 